\title{
Metaheuristic for the Capacitated Multiple Traveling Repairman Problem
}

\author{
Khanh-Phuong Nguyen ${ }^{1}$; Ha-Bang Ban ${ }^{2}$ \\ School of Information and Communication Technology \\ Hanoi University of Science and Technology, Hanoi, Vietnam \\ Corresponding authors: Ha-Bang Ban
}

\begin{abstract}
The Capacitated Multiple Traveling Repairmen Problem (CmTRP) is an extension of the Multiple Traveling Repairmen Problem (mTRP). In the CmTRP, the number of vehicles is dispatched to serve a set of customers, while each vehicle's capacity is limited by a predefined-value as well as each customer is visited exactly once. The goal is to find a tour that minimizes the sum of waiting times. The problem is NP-hard because it is harder than the mTRP. Even finding a feasible solution is also NP-hard problem. To solve medium and large size instances, a metaheuristic algorithm is proposed. The first phase constructs a feasible solution by combining between the Nearest Neighborhood Search (NNS) and Variable Neighborhood Search (VNS), while the optimization phase develops the feasible solution by the General Variable Neighborhood Search (GVNS). The combination maintains the balance between intensification and diversification to escape local optima. The proposed algorithm is implemented on benchmark instances from the literature. The results indicate that the developed algorithm obtains good feasible solutions in a short time, even for the cases with up to 200 vertices.
\end{abstract}

Keywords-CmTRP; NNS; VNS; GVNS

\section{INTRODUCTION}

\section{A. Motivation and Definition}

A particular variant of the CmTRP is the Multiple Traveling Repairmen Problem (mTRP) that considers multiple vehicles or travelers to find a tour minimizing the waiting time of all customers [1], [7], [9]. Applications of the MTRP can be found in [1], [7], [9]. The mTRP is based on an assumption that there is no limit to the capacity of each vehicle. That means vehicles can carry as many goods as they want. However, real situations imply that it does not always hold because vehicles have strict regulations on capacity. In this work, the mTRP is involved the capacity constraint. In the CmTRP, the maximum capacity of each vehicle does not exceed a predefined capacity $(Q)$. In the CmTRP, there are $k$ vehicles at a main depot $s$, and $n$ customers. The goal is to find a tour such that each vertex is visited exactly once, while the capacity constraint is satisfied, and the total waiting time of overall customers is minimized.

In this paper, we formulate the CmTRP as followings: We consider a complete graph $K_{n}$ that has the vertex set $V=$ $\{1,2, \ldots, n\}$ and a symmetric distance matrix $C=\{c(i, j) \mid$ $i, j=1,2, \ldots, n\}(c(i, j)$ is the traveling cost between vertex $i$ and vertex $j$ ). Each vertex $i$ has the demand $d_{i}$. Let $R=$ $(1,2, \ldots, k)$ be the number of $k$ vehicles which begin at a main depot $v_{1}$. Let $Q$ denote the capacity of a vehicle. Assume that a tour $T=\left(R_{1}, \ldots, R_{l}, \ldots, R_{k}\right)$ includes a set of routes from $k$ vehicles. $R_{l}=\left(v_{1}, \ldots, v_{h}, \ldots, v_{m}\right)(1<m \leq n)$ is a route of vehicle $l$. Let $P\left(v_{1}, v_{h}\right), l\left(P\left(v_{1}, v_{h}\right)\right)$ be respectively the path from vertex $v_{1}$ to vertex $v_{h}$ on $R_{l}$, and its length. The waiting time of $v_{h}(1<h \leq m)$ on $R_{l}$ is the cost of the path from $v_{1}$ to $v_{h}$ :

$$
l\left(P\left(v_{1}, v_{h}\right)\right)=\sum_{i=1}^{h-1} c\left(v_{i}, v_{i+1}\right) .
$$

Let $W\left(R_{l}\right)$ be the sum of waiting times of all vertices. The capacity of this route must satisfy the following constraint:

$$
\begin{gathered}
W\left(R_{l}\right)=\sum_{h=2}^{m} l\left(P\left(v_{1}, v_{h}\right)\right) . \\
D\left(R_{l}\right)=\sum_{i=1}^{m} d_{i} \leq Q .
\end{gathered}
$$

The objective function of the problem is:

$$
W(T)=\sum_{l=1}^{k} W\left(R_{l}\right) .
$$

The CmTRP asks for a tour, which starts at a depot $v_{1}$, visits each vertex once exactly with the waiting time of all vertices being minimized.

\section{B. Related Works}

In the general case, the CmTRP has, to the best of our knowledge, previously not been studied much, even though it is an extension of the mTRP case. However, some variants of the CmTRP are introduced in numerous works in the literature as follows:

- The mTRP is a particular case when the capacity constraint does not involve. Numerous works for mTRP can be found in [1], [7], [9]. In the metaheuristic approach, several algorithms [1], [7], [9] produce good solutions fast for instances with up to 200 vertices.

- The mTRP with distance constraint (mTRPD) is a particular case since the route length or maximum duration of each vehicle cannot exceed a predetermined limit. Metaheuristic algorithms in [3], [12] can solve the problem well for instances with up to 200 vertices.

- The mTRP with Profits (mTRPP) aims is to find a travel plan for server maximizing the total revenue. In this problem, not all customers need to be visited. Metaheuristic algorithm in [11] solves the problem well with up to 200 vertices. 
- The Traveling Repairman Problem is a special case where there is only a repairman. Numerous metaheuristic algorithms [2], [4], [5], [17] for the problem have proposed in the literature.

The above algorithms are the state-of-the-art algorithms for some variants of the CmTRP case. However, they do not include the capacity constraint, and their corresponding algorithms cannot be adapted to the CmTRP. That means that we cannot use the above algorithms to solve the CmTRP.

\section{Our Methodology}

Like other NP-hard problems, the CmTRP can be solved by exact or heuristic methods. Exact algorithms obtain the optimal solution but they often take exponential time in the worst case. Heuristic approaches are divided into the classical heuristic and metaheuristic approach. The classical heuristic approach finds one solution quickly, but this solution may have a large disparity in comparison with the best solution. The metaheuristic approach, on the other hand, obtains near-optimal or even global optimal solutions. Therefore, metaheuristic is usually used to reach optimal solutions for the problem with large sizes.

The CmTRP is also NP-hard because it is a generalization case of the mTRP. In many constrained optimization problems like the CmTRP, even building a feasible solution to the problem is also NP-hard problem. It indicates that obtaining a good feasible solution is a challenge. A good metaheuristic needs to ensure the balance between diversification and intensification. In [14], H. Mladenovic et al. show that the VNS generates local optima that are close to the global optimum, in a more straightforward manner than the other metaheuristics. However, the VNS only implements the intensification well. In this work, we developed a metaheuristic consisting of the constructive and optimization phase. The first phase constructs a feasible solution by combining between the Nearest Neighborhood Search (NNS) and Variable Neighborhood Search (VNS), while the optimization phase develops the feasible solution by the General Variable Neighborhood Search (GVNS). The metaheuristic uses the shaking technique to maintain diversification, while the VNS, and GVNS implement intensification. Extensive numerical experiments on benchmark instances show that the proposed algorithm reaches good feasible solutions at a reasonable amount of time, even for the instances with up to 200 vertices.

The rest of this paper is organized as follows. Section 2 introduces our algorithm. Computational evaluations are presented in Section 3, and Sections 4 and 5 discusses and concludes the work, respectively.

\section{The Proposed Algorithm}

\section{A. Several Variants of VNS}

We describe some variants of VNS [14] such as the original VNS, VND, and GVNS, shaking technique [13], respectively.

- $\quad$ The Variable Neighborhood Search (VNS) algorithm is introduced by Mladenovic et al. [14]. It executes neighborhood procedures alternately, and shaking technique to escape from the local optima. At each iteration, the best neighboring solution is chosen from neighboring solutions that are generated from a neighborhood procedure. If it is better than the current best one, the procedure is repeated. Otherwise, the search goes to the next neighborhood procedure.

- The Variable Neighborhood Descent (VND) algorithm, which is a VNS variant, is proposed by Mladenovic et al. [14]. In the VND, a change of neighborhoods is performed in a deterministic way. Assume that an initial solution is given. Local search procedures in their descent phase are used to generate neighborhoods. The final solution is the best solution in all neighborhoods. The difference between the VNS and VND is that the VNS uses Shaking.

- The General Variable Neighborhood Search (GVNS) algorithm [14] is a variant of the VNS. It includes an initial feasible solution, and a shaking procedure followed by the VND local search. The GVNS is a VNS variant where the VND is used as the improvement procedure.

\section{B. Neighborhoods}

We use seven neighborhoods in the literature to explore the search space of the problem. Let $N_{k}\left(k=1, \ldots, k_{m}\right)$ be a finite set of pre-selected neighborhood structures. We describe more details about seven neighborhoods:

For Inter-route: It is used to optimize on each route. We then describe five neighborhoods' structure in turn. Assume that, $R$, and $m$ are a route and its size, respectively.

- Remove-insert places each vertex in the route at the end of it. Obviously, the complexity time of $N_{1}(R)$ is $O(m)$.

- Swap adjacent tries to swap each pair of adjacent vertices in the route. The complexity time of $N_{2}(R)$ is $O(m)$.

- Swap neighborhood attempts to swap the positions of each pair of vertices in the route. The complexity time of $N_{3}(R)$ is $O\left(m^{2}\right)$.

- 3-opt neighborhood attempts to reallocate three vertices to another position of the route. The complexity time of $N_{4}(R)$ is $O\left(m^{3}\right)$.

- 4-opt neighborhood attempts to involve deleting four edges and reconnecting the four sub-tours without changing the orientation of them. The complexity time of $N_{5}(R)$ is $O\left(m^{4}\right)$.

For intra-route: Intra-route is used to swap vertices between two different routes or remove vertices from a route and then insert them to another.Let $R_{l}, R_{h}, m l$, and $m h$ be two different routes and their sizes in $T$, respectively.

- $\quad$ swap-2-routes tries to exchange two vertices belonging to different routes $R_{l}$ and $R_{h}$. The complexity time of $N_{6}(R)$ is $O(m l \times m h)$

- insert-2-routes removes one vertex $R_{l}$ and inserts it at the best possible position in $R_{h}$. The complexity time of $N_{7}(R)$ is $O(m l \times m h)$. 

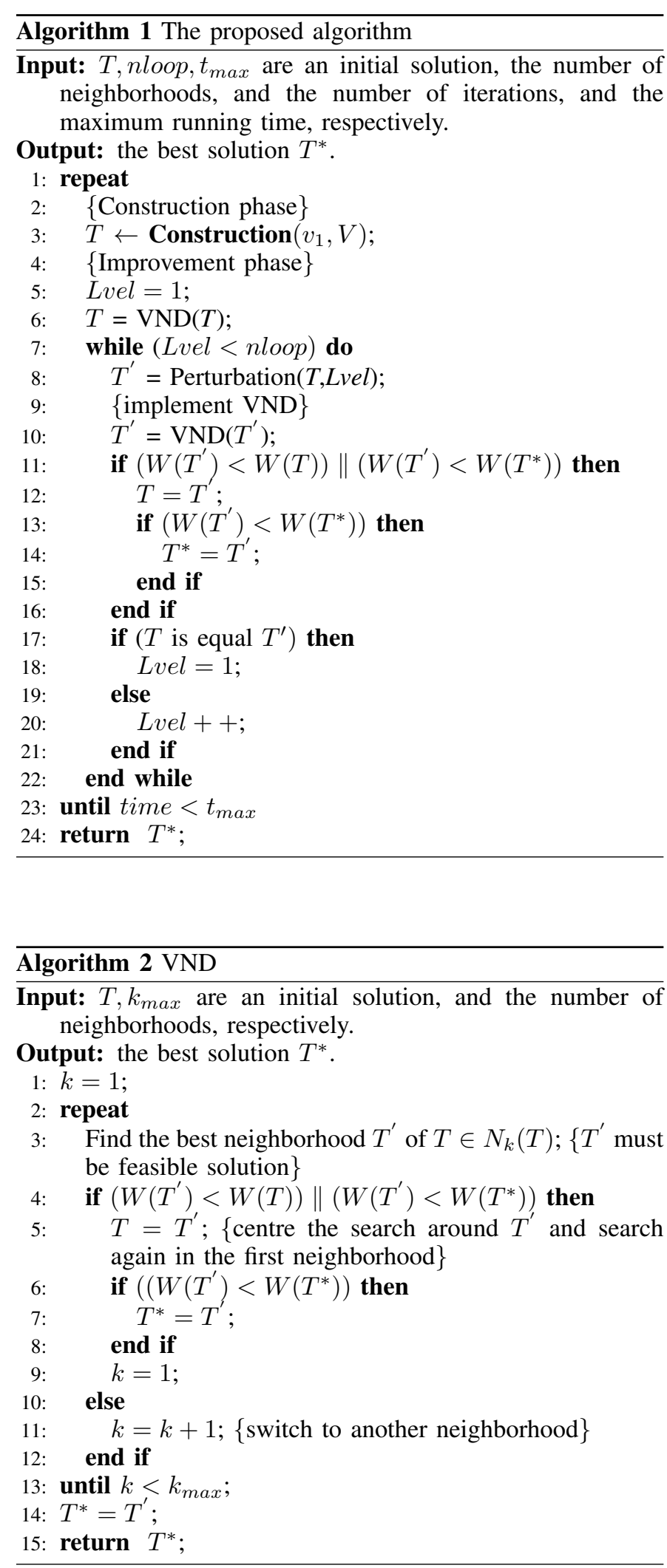

The proposed algorithm includes two phases. The construction phase finds a feasible solution, whereas the improvement phase tries to improve it. Algorithm 1 depicts the whole process.
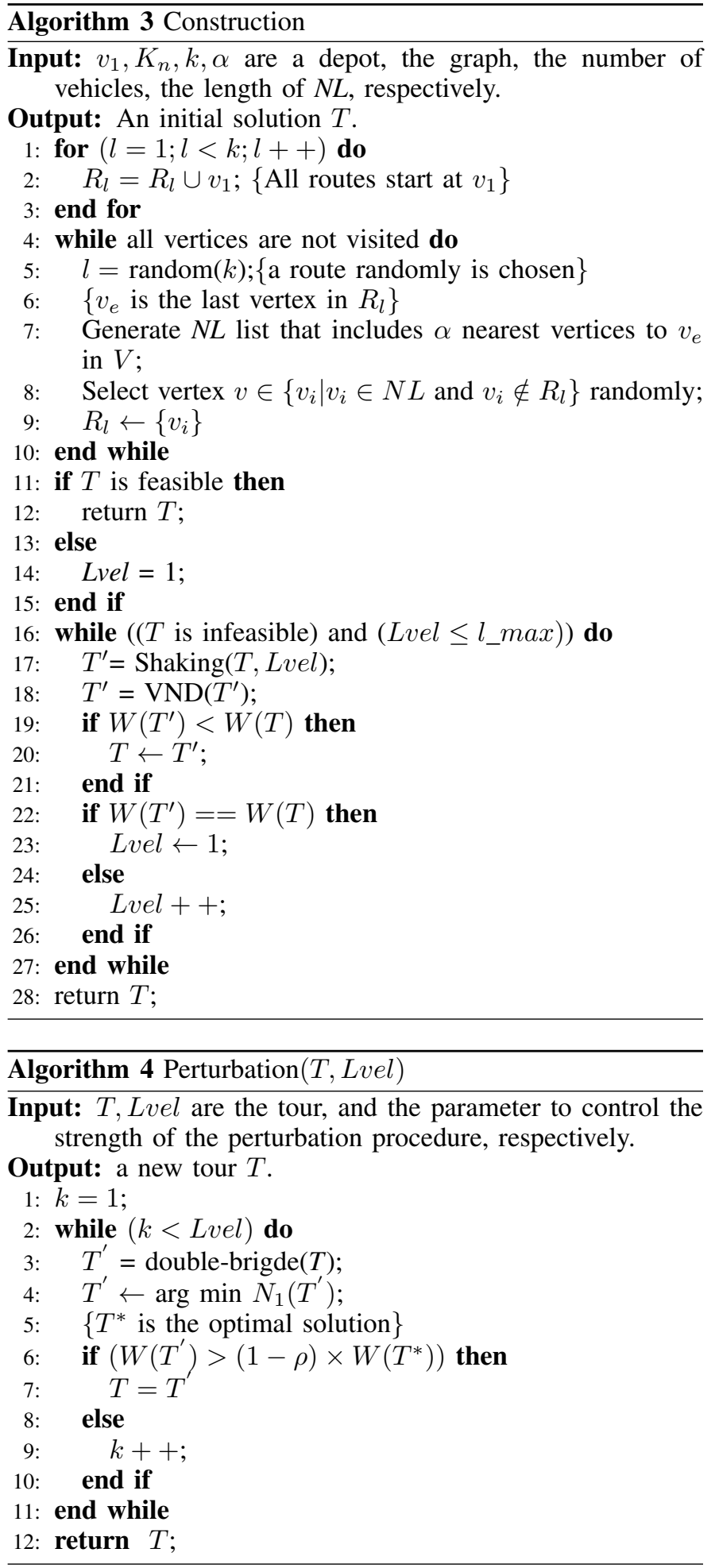

\section{Construction}

Algorithm 2 shows the constructive procedure. The objective function used in this procedure is the sum of all positive differences between the capacity of all vehicles and the capacity limit $Q$, that is, $\min \sum_{l=1}^{k} \max \left(0, D\left(R_{l}\right)-Q\right)$. The algorithm works until it finds a feasible solution. In the first step, a solution is created by Nearest Neighborhood Search [8]. If the solution is feasible, the construction phase stops 
TABLE I. OUR RESULTS FOR THE CMTRP ON E-INSTANCES PROPOSED BY [18]

\begin{tabular}{|l|l|r|r|r|r|}
\hline Instances & Init.Sol & Best.Sol & Aver.Sol & Improv & $T$ \\
\hline E30k3 & 3008.64 & 2419.1 & 2419.1 & 19.59 & 2 \\
\hline E30k4 & 2052.97 & 1731.43 & 1731.43 & 15.66 & 3 \\
\hline E51k5 & 2948.73 & 2769.1 & 2769.1 & 6.09 & 7 \\
\hline E76k10 & 3301.85 & 3064.68 & 3064.68 & 7.18 & 27 \\
\hline E76k14 & 2409.68 & 2261.63 & 2261.63 & 6.14 & 14 \\
\hline E76k15 & 2401.86 & 2221.59 & 2221.59 & 7.51 & 17 \\
\hline aver & & & & 10.36 & 11.7 \\
\hline
\end{tabular}

TABLE II. OUR RESULTS FOR THE CMTRP ON P-INSTANCES PROPOSED BY [18]

\begin{tabular}{|l|r|r|r|r|r|}
\hline Instances & Init.Sol & Best.Sol & Aver.Sol & Improv & $T$ \\
\hline P40k5 & 2095.22 & 1884.55 & 1884.55 & 10.05 & 5 \\
\hline P45k5 & 2672.9 & 2479.41 & 2479.41 & 7.24 & 8 \\
\hline P50k7 & 2262.5 & 1993.24 & 1993.24 & 11.90 & 7 \\
\hline P50k8 & 2310.09 & 2310.09 & 2310.09 & 0.00 & 4 \\
\hline P55k7 & 2325.41 & 2175.46 & 2175.46 & 6.45 & 13 \\
\hline P55k10 & 2095.43 & 1878.64 & 1878.64 & 10.35 & 6 \\
\hline P60k10 & 2511.53 & 2322.96 & 2322.96 & 7.51 & 15 \\
\hline P70k10 & 3297.38 & 2940.94 & 2940.94 & 10.81 & 17 \\
\hline P76k4 & 6509.49 & 6198.5 & 6198.5 & 4.78 & 28 \\
\hline P76k5 & 5920.72 & 5637.31 & 5637.31 & 4.79 & 29 \\
\hline aver & & & & 7.39 & 13.2 \\
\hline
\end{tabular}

TABLE III. OUR RESULTS FOR THE CMTRP ON TAI-INSTANCES PROPOSED BY [18]

\begin{tabular}{|l|r|r|r|r|r|}
\hline Instances & \multicolumn{1}{|c|}{ Init.Sol } & Best.Sol & Aver.Sol & Improv & $T$ \\
\hline tai75a & 7038 & 6286.77 & 6331.913 & 10.67 & 32 \\
\hline tai75b & 6112.16 & 5061.1 & 5156.406 & 17.20 & 30 \\
\hline tai75c & 5517.07 & 5023.56 & 5085.652 & 8.95 & 35 \\
\hline tai75d & 6347.94 & 5856.79 & 5974.241 & 7.74 & 37 \\
\hline tai100a & 11391.44 & 10200.9 & 10290.74 & 10.45 & 72 \\
\hline tai100b & 10297.75 & 9755.82 & 9801.026 & 5.26 & 73 \\
\hline tai100c & 6025.94 & 5743.6 & 5792.192 & 4.69 & 70 \\
\hline tai100d & 7544.23 & 7138.13 & 7203.689 & 5.38 & 68 \\
\hline tai150a & 16435.68 & 15913.87 & 16005.03 & 3.17 & 85 \\
\hline tai150b & 14115.32 & 13627.56 & 13687.99 & 3.46 & 81 \\
\hline tai150c & 14797.07 & 13098.63 & 13208.41 & 11.48 & 82 \\
\hline tai150d & 15268.64 & 13530.54 & 13530.54 & 11.38 & 83 \\
\hline aver & & & & 8.32 & 62.3 \\
\hline
\end{tabular}

and outputs it. On the other hand, a local search iterates until finding a feasible solution or $l_{-} \max$ is reached. The solution is shaken to escape from the current local optimal. Next, the VNS is applied to obtain the best solution from neighboring solutions. If it is better than the found best solution, it is set to the current solution. Last, Lvel is increased by one if the current solution is not improved, or set to 1 , otherwise.

\section{Improvement}

After the construction, the heuristic tries to improve the feasible solution created by the previous phase. In this phase, the objective function is to minimize $W(T)$.

Local search procedure that is developed by combining the seven neighborhoods generates various neighborhoods. The final solution should be a local minimum with respect to all neighborhoods. The order of neighborhoods is fixed.
In a preliminary experiment, the other of the neighborhoods are therefore explored in the following one, from "small" to "large" as it is common, i.e., swap-adjacent, move-up, movedown, remove-insert, swap, 2-opt, 3-opt, 4-opt, swap-2-routes, and insert-2-routes.

The Perturbation mechanism is very important to achieve success. When the mechanism has too small perturbation moves, the search can return to the previously visited solution space. Therefore, the search can get stuck into local optimal. On the other hand, large perturbation moves drive the search to undesirable space. In order to overcome these issues, we propose a new shaking technique based on the original doublebridge technique [13]. The detail is described in Algorithm 3.

The algorithm stops after $t_{\max }$ seconds or the best-solution is found $\left(t_{\max }\right.$ is the parameter of the algorithm, and its value is determined from preliminary experiments).

\section{EVALUATIONS}

The proposed algorithm is run on a Pentium 4 core i7 $2.40 \mathrm{GHz}$ processor with $8 \mathrm{~GB}$ of RAM. On all experiments, parameters $\alpha, l_{\_} \max , \rho$, nloop are respectively set to 10,5 , 0.3 , and 50 . These parameters are chosen through empirical tests and, with them, the algorithm seems to produce good solutions at a reasonable amount of time in comparison with the other parameter values.

We also implement the performance of the whole implementation against the state-of-the-art algorithms. We compare both of the numerical results and computational time on the same instances.

\section{A. Instances}

The experiments are performed on a set of benchmark for Capacitated VRP in [12], [18]. As testing the proposed algorithm on overall instances can be computationally too expensive, some selected instances as follows: 1) to eliminate the effects of size, instances with approximately from 50 up to 200 customers are chosen; 2) in order not to bias the results by taking "easy" or "hard" instances, we randomly select them. These are: 1) Christofides et al.: This dataset includes seven instances (CMT6, CMT7, ..., CMT14); 2) Taillard et al.: Ten instances are picked randomly such as tai75a, tai75b, tai75c, tai75d, tai100a, tai100b, tai100c, tai100d, tai150a, tai150b, tai150c, and tai150d; 3) Augerat et al.: Fifteen instances of dataset $P$ and $E$ are selected; 4) S. Nucamendi-Guillén et al.: 150 instances from 60 to 80 vertices are used in the experiments. The optimal solutions for the instances can be extracted from [16].

\section{B. Results}

We define the improvement of our algorithm with respect to Best.Sol (Best.Sol is the best solution found by the proposed algorithm) in comparison with the initial solution from the construction phase as followings:

$$
\text { Improv }[\%]=\frac{\text { Best.Sol }- \text { Init.Sol }}{\text { Init.Sol }} \times 100 \%
$$

In the tables, OPT, Init.Sol, Best.Sol, Aver.Sol and $T$ correspond to the optimal solution, initial solution, best solution, average 
(IJACSA) International Journal of Advanced Computer Science and Applications,

Vol. 11, No. 5, 2020

TABLE IV. THE EVOLUTION OF AVERAGE IMPROVEMENT DURING THE ITERATIONS

\begin{tabular}{|l|c|c|c|c|c|c|c|c|c|c|c|c|c|c|}
\hline \multicolumn{1}{|c|}{ instances } & \multicolumn{3}{|c|}{1 iteration } & \multicolumn{2}{|c|}{10 iterations } & \multicolumn{2}{c|}{20 iterations } & \multicolumn{2}{c|}{30 iterations } & \multicolumn{2}{c|}{40 iterations } & \multicolumn{2}{c|}{50 iterations } & \multicolumn{2}{c|}{100 iterations } \\
\hline E-instance & 6.85 & 0.2 & 7.65 & 1.48 & 8.45 & 4.32 & 9.78 & 6 & 10.1 & 8.17 & 10.4 & 11.7 & 10.4 & 17.33 \\
\hline P-instance & 5.5 & 0.2 & 5.8 & 1.7 & 6 & 4.9 & 6.4 & 6.8 & 6.8 & 9.2 & 7.4 & 13.2 & 7.4 & 25.9 \\
\hline Tai-instances & 6.7 & 1.1 & 7.1 & 7.9 & 7.4 & 23.0 & 7.8 & 31.9 & 8 & 43.5 & 8.3 & 62.3 & 8.3 & 187.9 \\
\hline aver & 6.35 & 0.50 & 6.85 & 3.68 & 7.28 & 10.73 & 7.99 & 14.91 & 8.30 & 20.30 & 8.70 & 29.07 & 8.70 & 77.04 \\
\hline
\end{tabular}

TABLE V. THE DIFFERENCE BETWEEN THE CMTRP AND MTRP'S OBJECTIVE FUNCTION ON SOME INSTANCES

\begin{tabular}{|l|c|r|r|}
\hline Instances & mTRP & CmTRP & \%diff \\
\hline E30k3 & 1871.08 & 2419.1 & 29.29 \\
\hline E30k4 & 1643.30 & 1731.43 & 5.36 \\
\hline E51k5 & 2209.64 & 2769.1 & 25.32 \\
\hline E76k10 & 2310.09 & 3064.68 & 32.66 \\
\hline E76k14 & 2005.40 & 2261.63 & 12.78 \\
\hline E76k15 & 1962.47 & 2221.59 & 13.20 \\
\hline P40k5 & 1537.79 & 1884.55 & 22.55 \\
\hline P45k5 & 1912.31 & 2479.41 & 29.66 \\
\hline P50k7 & 1547.89 & 1993.24 & 28.77 \\
\hline aver & & & 22.18 \\
\hline
\end{tabular}

solution, and the average time in seconds of ten executions obtained by our algorithm, respectively. In this work, we choose several state-of-the-art metaheuristic algorithms for some variants of the CmTRP [1], [6], [10], [12], [15], [16] as a baseline in our research.

Tables I to III show the average gap of the improvement phase in comparison with the construction is $8.70 \%$. The average gap value is not too large. This indicates that the construction phase gives good feasible solutions fast. The proposed algorithm consumes much time for the instances with up to 200 vertices. Therefore, the fist way to decrease the running time is only to run the construction phase. In this case, the proposed algorithm suffers from a slightly loss of $8.70 \%$ solution quality on average. Although we cannot compare the results directly to other algorithms in the literature (note that in the algorithms for some variants of the mTRP, the capacity constraint is removed), we succeed in producing feasible solutions for instances with 200 customers. It is an important contribution when finding feasible solutions is also NP-hard.

Table IV shows the evolution of average gap during the iterations. The average gaps are $6.35 \%, 6.85 \%, 7.28 \%, 7.99 \%$, $8.30 \%, 8.70 \%$, and $8.70 \%$ in comparison with the initial solution, obtained by one, five, ten, twenty, thirty, forty, fifty, and one-hundred iterations, respectively. No improvement obtains from fifty to one-hundred iterations. Therefore, additional iterations give a minor improvement while it consumes much time. Hence, the second way to reduce the running time is to use no more than fifty iterations, and the improvement reaches about $8.70 \%$. The fastest option is to run the construction phase and then improve it by using a single iteration, which obtains an average gap of $6.35 \%$ and average time of 0.5 seconds.
TABLE VI. OUR RESULTS FOR THE MTRP-INSTANCES WITH 60 VERTICES PROPOSED BY LUO

\begin{tabular}{|c|c|c|c|c|c|}
\hline Instances & $O P T$ & Init.Sol & Best.Sol & Aver:Sol & $T$ \\
\hline pr1002_60_0 & 530946.01 & 660211.35 & 530946.01 & 530946.01 & 2.98 \\
\hline pr1002_60_1 & 56469.79 & 455893.41 & 356469.79 & 356469.79 & 2.84 \\
\hline pr1002_60_2 & 344118.14 & 467498.53 & 344118.14 & 344118.14 & 2.98 \\
\hline pr1002_60_3 & 429604.2 & 579392.35 & 429604.2 & 429604.2 & 2.92 \\
\hline pr1002_60_4 & 435655.25 & 540342.11 & 435655.25 & 435655.25 & 2.82 \\
\hline pr1002_60_5 & 668129.73 & 779776.11 & 668129.73 & 668129.73 & 2.86 \\
\hline pr1002_60_6 & 406678.77 & 495022.53 & 406678.77 & 406678.77 & 2.9 \\
\hline pr1002_60_7 & 31125 & & 311254.73 & & 2.98 \\
\hline pr1002_60_8 & 469816.84 & & 4698 & & 3 \\
\hline pr1002_60_9 & & & 2773 & & 2.84 \\
\hline 051600 & & 26789 & & & 3 \\
\hline brd14051_60_1 & & & & & 2.98 \\
\hline 051602 & & & & & 2.9 \\
\hline 1603 & & & & & 2.96 \\
\hline $1 \_60 \_4$ & & 608 & & & 2.84 \\
\hline 1605 & & 286 & & & 2.88 \\
\hline 1 160_6 & & & & & 2.98 \\
\hline $60 \quad 7$ & & & 171 & & 2.96 \\
\hline 1608 & & 82 & & & 2.98 \\
\hline 1_60_9 & & 25 & & & 2.94 \\
\hline $60 \_0$ & & 83 & 156 & & 2.8 \\
\hline 601 & & 61 & 103 & & 2.96 \\
\hline 602 & & & 109 & & 2.98 \\
\hline $1 \_603$ & & 575 & & & 2.94 \\
\hline 16044 & & 777 & 149 & & 2.96 \\
\hline $61 \_60 \_5$ & & 379 & 158 & & 2.96 \\
\hline fn14461_60_6 & 6.92 & 283 & 1222 & 6.92 & 2.88 \\
\hline fnl4461_60_7 & & 532 & 107 & 9.11 & 2.94 \\
\hline fn14461_60_8 & & 749 & 1.72 & 1.72 & 2.84 \\
\hline fnl4461_60_9 & .76 & 156 & 9.76 & 9.76 & 2.94 \\
\hline $12 \_60 \_0$ & & 482 & .42 & 9.42 & 2.8 \\
\hline $1260 \quad 1$ & & 493 & .99 & 9.99 & 2.86 \\
\hline $12 \_60 \_2$ & & 404 & .33 & 9.33 & 2.82 \\
\hline $12 \_60 \_3$ & & & .95 & 7.95 & 2.82 \\
\hline $260 \_4$ & & & 82 & & 2.96 \\
\hline $60 \_5$ & & 263 & 422 & & 2.94 \\
\hline 606 & & & & & 2.86 \\
\hline 607 & & & 44 & & 2.98 \\
\hline 608 & & 875 & 9.37 & 9.37 & 2.8 \\
\hline 609 & .81 & 184 & 673 & 0.81 & 2.9 \\
\hline nrw1379 $60 \_0$ & .77 & 654 & 9.77 & 9.77 & 2.88 \\
\hline nrw1379_60_1 & .67 & 1046 & 0.67 & 83410.67 & 2.96 \\
\hline nrw1379_60_2 & 8.87 & 333 & 52858.87 & 52858.87 & 2.96 \\
\hline nrw1379 $60 \quad 3$ & .36 & 0476 & 1.36 & 62341.36 & 2.84 \\
\hline nrw1379_60_4 & 56012.13 & 69680 & 56012.13 & 56012.13 & 2.9 \\
\hline nrw1379_60_5 & 083.8 & 3.325 & 58083.8 & 58083.8 & 2.9 \\
\hline nrw1379_60_6 & 52224.66 & 65749.025 & 52224.66 & 52224.66 & 2.92 \\
\hline nrw1379_60_7 & 58402.97 & 73290.6375 & 58402.97 & 58402.97 & 2.94 \\
\hline nrw1379_60_8 & 52145.08 & 66101.85821 & 52145.08 & 52145.08 & 2.96 \\
\hline nrw1379_60_9 & 49026.52 & 66572.84307 & 49026.52 & 49026.52 & 2.86 \\
\hline
\end{tabular}

Table V shows the difference between the objective function of two problems on the same instances. The average

difference of $22.18 \%$ indicates the capacity constraint also 
(IJACSA) International Journal of Advanced Computer Science and Applications,

Vol. 11, No. 5, 2020

TABLE VII. OUR RESULTS FOR THE MTRP-INSTANCES WITH 70 VERTICES PROPOSED BY LUO
TABLE VIII. OUR RESULTS FOR MTRP-INSTANCES WITH 80 VERTICES PROPOSED BY LUO

\begin{tabular}{|c|c|c|c|c|c|}
\hline Instances & $O P T$ & Init.Sol & Best.Sol & Aver:Sol & $T$ \\
\hline pr1002_70_0 & 429557.7 & 429557.7 & 429557.7 & 530946.01 & 3.12 \\
\hline pr1002 $70 \_1$ & 30048.06 & 430048.06 & 430048.06 & 356469.79 & \\
\hline pr1002 $70 \_2$ & 7233.86 & 377233.86 & 377233.86 & & 2.68 \\
\hline pr1002_70_3 & 9562.01 & 429562.01 & 429562.01 & 429604.2 & 2.64 \\
\hline pr1002 704 & 5659.17 & 435659.17 & 435659.17 & 435655.25 & 2.96 \\
\hline pr1002 705 & 29584.16 & 429584.16 & 429584.16 & 68129.73 & 3.34 \\
\hline pr1002_70_6 & 44534.44 & 3445 & 3445 & 406678.77 & 2.82 \\
\hline pr1002 $70 \quad 7$ & 3558.46 & 3935 & 393 & & 3.02 \\
\hline pr1002 708 & 7072.39 & 3970 & & & 2.74 \\
\hline pr1002_70_0 & 4295 & & & & 3.12 \\
\hline brd14051_70_0 & 191843.35 & 1918 & & & 2.76 \\
\hline $170 \_1$ & 169340.01 & & & & 2.98 \\
\hline 1702 & 216195.95 & 2161 & & & 3.12 \\
\hline 1703 & & & & & 3.28 \\
\hline 704 & & & & & 3.34 \\
\hline 705 & & & & & \\
\hline 706 & & & & & 2.66 \\
\hline 707 & & & & & 66 \\
\hline $70 \_8$ & & & & & 76 \\
\hline 709 & & & & & 26 \\
\hline 700 & & & & & 2.76 \\
\hline $70 \_1$ & & & & & 3.24 \\
\hline 702 & & & & & 2.74 \\
\hline 1703 & & & & & 3.32 \\
\hline 1704 & .13 & 3 & & & 2.84 \\
\hline 1705 & 64 & 112 & & & 2.72 \\
\hline 1 1_70_6 & & & & & 2.74 \\
\hline $61 \_70 \_7$ & & 51 & & & 3.06 \\
\hline fnl4461_70_8 & & & & & 2.94 \\
\hline 1 1_70_0 & & & & & 2.76 \\
\hline $70 \_0$ & & & & & 3.24 \\
\hline 701 & & & & & 3.02 \\
\hline 702 & & & & & \\
\hline 703 & & & & & 3.32 \\
\hline 704 & & & & & 2.78 \\
\hline 705 & & & & & 3.18 \\
\hline $70 \_6$ & 9.6 & & & & 3.18 \\
\hline 707 & 85 & & & & 2.86 \\
\hline 708 & 51 & & & & 3.02 \\
\hline 709 & 6.3 & & & & 2.6 \\
\hline 700 & & & & & 2.58 \\
\hline 701 & & & & & 2.98 \\
\hline $70 \_2$ & 0.7 & & & 3.87 & 3.2 \\
\hline 9703 & & & & & 3.32 \\
\hline 9704 & 594.56 & & & 2.13 & 2.66 \\
\hline nrw1379_70_5 & 73884.17 & & 17 & 83.8 & 3.02 \\
\hline nrw1379 $70 \quad 6$ & 306.14 & & 6.14 & 52224.66 & 2.94 \\
\hline nrw1379_70_7 & 5554.87 & & & 58402.97 & 2.54 \\
\hline nrw1379_70_8 & 91738.43 & & 91738.43 & 52145.08 & 2.82 \\
\hline nrw1379 709 & 68024.3 & 68024.3 & 68024.3 & 49026.52 & 2.68 \\
\hline
\end{tabular}

affects the quality of solutions. However, the best solutions for the mTRP are not feasible solutions for the CmTRP. Therefore, the methods designed for the mTRP instances may not be adapted easily to solve the CmTRP.

From Tables VI to VIII show that the efficiency of the proposed algorithm is very good for the mTRP-instances since it can reach the optimal solutions for the instances with up to 80 vertices at a reasonable amount of time. In Table IX, in comparison with Ban et al.'s [1], Ezzine et al.'s [6], and Nucamendi-Guillén's [16] algorithm, our solutions are better than those of Ban et al.'s, and Ezzine et al. in all cases while

\begin{tabular}{|c|c|c|c|c|c|}
\hline Instances & $O P T$ & Init.Sol & Best.Sol & Aver:Sol & $T$ \\
\hline pr1002 $80 \quad 0$ & 491764.64 & 656239.68 & 491764.64 & 491764.64 & 9.48 \\
\hline pr1002 $80 \quad 1$ & 442164.21 & 613287.46 & 442164.21 & 442164.21 & 9.18 \\
\hline pr1002 802 & 505524.17 & 609954.56 & 505524.17 & 505524.17 & 9.32 \\
\hline pr1002 803 & 436752.96 & 614611.29 & 436752.96 & & 9.1 \\
\hline pr1002 804 & 453609.46 & 587470.83 & 453609.46 & 453609.46 & 9.36 \\
\hline pr1002 80_5 & 599492.4 & 771733.95 & 599492.4 & 599492.4 & 9.16 \\
\hline pr1002_80_6 & 619003.36 & 805206.35 & 619003.36 & 619003.36 & 9.4 \\
\hline pr1002 807 & 508186.51 & 658640.85 & 508186.51 & 508186.51 & 9.42 \\
\hline pr1002_80_8 & 409733.88 & 518052.51 & 409733.88 & 409733.88 & 9.44 \\
\hline pr1002 809 & 557220.48 & 670387.49 & 557220.48 & 557220.48 & 9.28 \\
\hline brd14051 800 & 336983.07 & 403178.34 & 336983.07 & 336983.07 & 9.04 \\
\hline brd14051 $80 \quad 1$ & 277861.02 & 348787.38 & 277861.02 & 277861.02 & 9.14 \\
\hline brd14051_80_2 & 265370.92 & 321922.47 & 265370.92 & 265370.92 & 9.56 \\
\hline brd14051 $80 \quad 3$ & 189815.69 & 240361.74 & 189815.69 & 189815.69 & 9.1 \\
\hline brd14051_80 4 & 206068.45 & 275228.43 & 206068.45 & 206068.45 & 9.5 \\
\hline brd14051_80_5 & 303621.75 & 348578.27 & 303621.75 & 303621.75 & 9.32 \\
\hline brd14051_80_6 & 213405.23 & 266958.37 & 213405.23 & 213405.23 & 9.6 \\
\hline brd14051_80_7 & 263737.93 & 308039.16 & 263737.93 & 263737.93 & 9.04 \\
\hline brd14051 808 & 232967.83 & 298574.84 & 232967.83 & 232967.83 & 9.26 \\
\hline brd14051 809 & 317790.55 & 368183.51 & 317790.55 & 317790.55 & 9.06 \\
\hline fn14461_80_0 & 153124.51 & 194685.8 & 153124.51 & 153124.51 & 9.58 \\
\hline fn14461_80_1 & 174975.64 & 224516.74 & 174975.64 & 174975.64 & 9 \\
\hline fnl4461_80_2 & 162755.5 & 197782.39 & 162755.5 & 162755.5 & 9.46 \\
\hline fnl $4461 \quad 80 \quad 3$ & 160819.04 & 192927.87 & 160819.04 & 160819.04 & 9.5 \\
\hline fnl $4461 \quad 80 \quad 4$ & 151790.69 & 187440.09 & 151790.69 & 151790.69 & 9.52 \\
\hline fnl4461 805 & 131045.47 & 172293.83 & 131045.47 & 131045.47 & 9.04 \\
\hline fnl 4461806 & 125405.93 & 166418.99 & 125405.93 & 125405.93 & 9.24 \\
\hline $\begin{array}{llll}\text { fnl } 4461 & 80 & 7\end{array}$ & 125228.91 & 164627.71 & 125228.91 & 125228.91 & 9.16 \\
\hline fnl4461 $80 \quad 8$ & 185280.87 & 228208.31 & 185280.87 & 185280.87 & 9.48 \\
\hline fnl4461_80 9 & 130304.95 & 165022.1 & 130304.95 & 130304.95 & 9.26 \\
\hline d15112_80_0 & 551900.43 & 753989.49 & 551900.43 & 551900.43 & 9.56 \\
\hline d15112_80_1 & 815029.39 & 979921.76 & 815029.39 & 815029.39 & 9.1 \\
\hline d15112_80_2 & 828114.32 & 1080571.45 & 828114.32 & 828114.32 & 9.16 \\
\hline d15112_80_3 & 689450.94 & 964458.54 & 689450.94 & 689450.94 & 9.1 \\
\hline d15112_80_4 & 560385.47 & 737417.48 & 560385.47 & 560385.47 & 9.08 \\
\hline d15112_80_5 & 821959.4 & 1030515.79 & 821959.4 & 821959.4 & 9.52 \\
\hline d15112_80_6 & 715206.03 & 882086.8 & 715206.03 & 715206.03 & 9.36 \\
\hline d15112_80_7 & 958278.86 & 1155190.13 & 958278.86 & 958278.86 & 9.32 \\
\hline d15112_80_8 & 990277.77 & 1174384.17 & 990277.77 & 990277.77 & 9.1 \\
\hline $\mathrm{d} 15112809$ & 672457.47 & 931587.71 & 672457.47 & 672457.47 & 9.5 \\
\hline $\begin{array}{llll}\text { nrw1379 } & 80 & 0\end{array}$ & 64831.76 & 96656.39 & 64831.76 & 64831.76 & 9.36 \\
\hline nrw1379 $80 \_1$ & 64967.83 & 88394.72 & 64967.83 & 64967.83 & 9.22 \\
\hline nrw1379 $80 \quad 2$ & 73858.13 & 96499.97 & 73858.13 & 73858.13 & 9.3 \\
\hline nrw1379 $80 \quad 3$ & 100592.83 & 131733.34 & 100592.83 & 100592.83 & 9.24 \\
\hline nrw1379 $80 \quad 4$ & 98228.29 & 126451.61 & 98228.29 & 98228.29 & 9.04 \\
\hline nrw1379 805 & 75984.21 & 99492.47 & 75984.21 & 75984.21 & 9.14 \\
\hline nrw1379 $80 \quad 6$ & 79165.6 & 105024.23 & 79165.6 & 79165.6 & 9.08 \\
\hline nrw1379 $80 \quad 7$ & 73194.55 & 105328.52 & 73194.55 & 73194.55 & 9.1 \\
\hline nrw1379 808 & 83492.62 & 115793.31 & 83492.62 & 83492.62 & 9.14 \\
\hline $\begin{array}{llll}\text { nrw1379 } & 80 & 9\end{array}$ & 67034.31 & 92380.75 & 67034.31 & 67034.31 & 9.24 \\
\hline
\end{tabular}

they are comparable with Nucamendi-Guillén's solutions in the most of instances. In many cases, our algorithm obtains the optimal solution for the instance with 76 vertices. Moreover, for the CCVRP in Table X, our algorithm reaches the known best solutions for the instances with 100 vertices (note that: the best solutions are extracted from [10], [15]).

The running time of the proposed algorithm grows quite moderate compared to the Nucamendi-Guillén's algorithm [16] while it is comparable with those of Ban et al.'s [1], and Ezzine et al.'s algorithm [6]. 
TABLE IX. COMPARISONS WITH THE PREVIOUS ALGORITHMS FOR MTRP-INSTANCES PROPOSED IN [18]

\begin{tabular}{|l|c|c|c|r|c|}
\hline \multirow{2}{*}{ Instances } & \multirow{2}{*}{$\begin{array}{c}\text { Ban } \\
\text { et al. }\end{array}$} & \multirow{2}{*}{$\begin{array}{c}\text { Ezzine } \\
\text { et al. }\end{array}$} & $\begin{array}{c}\text { Nucamendi- } \\
\text { Guillén } \\
\text { et al. }\end{array}$ & \multicolumn{2}{|c|}{ Our results } \\
\cline { 5 - 6 } & & & Best.Sol & $T$ \\
\hline E30k3 & 2108.26 & - & - & 2097.3 & 0.25 \\
\hline E30k4 & 2623.65 & - & - & 2595.11 & 0.22 \\
\hline E51k5 & 2623.65 & 3320 & $2209.64^{*}$ & 2209.64 & 0.41 \\
\hline E76k10 & 2786.07 & 4094 & $2310.09^{*}$ & 2419.89 & 0.78 \\
\hline E76k14 & 2201.13 & 3762 & $2005.4^{*}$ & 2005.4 & 0.71 \\
\hline E76k15 & 2400.17 & & - & 2377.5 & 0.81 \\
\hline E101k8 & - & 6383 & - & 4051.47 & 2.52 \\
\hline E101k14 & - & 5048 & - & 3288.53 & 2.50 \\
\hline P40k5 & 1793.14 & - & $1537.79^{*}$ & 1580.21 & 0.27 \\
\hline P45k5 & 2336.43 & - & $1912.31 *$ & 1912.31 & 0.32 \\
\hline P50k7 & 1878.81 & - & $1547.89^{*}$ & 1590.41 & 0.58 \\
\hline \multicolumn{3}{r}{ Note that: symbol ‘*'is the optimal value } & \\
\end{tabular}

TABLE X. COMPARISONS WITH THE PREVIOUS ALGORITHMS FOR CCVRP-INSTANCES PROPOSED BY [18]

\begin{tabular}{|l|c|c|r|}
\hline Instances & BKS & Best.Sol & \multicolumn{1}{|c|}{$T$} \\
\hline CMT1 & 2230.35 & 2230.35 & 1.70 \\
\hline CMT2 & 2391.63 & 2429.18 & 6.19 \\
\hline CMT3 & 4045.42 & 4073.12 & 17.93 \\
\hline CMT4 & 4987.52 & 4987.52 & 85.94 \\
\hline CMT5 & 5809.59 & 5838.32 & 295.67 \\
\hline CMT11 & 7314.55 & 7314.55 & 31.09 \\
\hline CMT12 & 3558.92 & 3559.43 & 15.84 \\
\hline
\end{tabular}

\section{Discussions}

Due to NP-hard problem, metaheuristic approach is suitable approach to solve the problem with large sizes in a short time. Currently, several algorithms for the close variants of CmTRP have been proposed. However, these algorithms cannot apply to the CmTRP because the capacity constraint does not include in them. The results in Table $\mathrm{V}$ indicate that the algorithms for the mTRP produce infeasible solutions for the CmTRP. Therefore, developing efficient algorithm for the CmTRP is our contribution. The VNS, and GVNS [14] are a general schemes that are used widely to solve many optimization problems. However, to apply them for a specific problem, they require many efforts. Specifically, how to use and combine neighborhoods to explore and exploit good solution space, how many neighborhoods are used to balance between solution quality and running time, and how to balance between diversity and intensification. Therefore, in the literature, many algorithms are developed on the VNS scheme for a specific problem, but the solution quality of them is different. Applying the VNS scheme to solve the problem effectively is our contribution in this work.

For a metaheusitic approach, a classical and successful recipe is to combine 1) efficient "intensification" procedures via local searches with 2) "diversification" methods. Diversification means to generate diverse solutions to explore the search space on a global scale, while intensification means to focus on the search in a local region by exploiting the information that a current good solution is found in this region. Our algorithm uses the VNS, GVNS to implement intensification while shaking maintains diversification. Though the initial solution is generated far from the global minima, the explored solution region is extended. Therefore, the chance for finding good solutions is high. The CmTRP is harder than the mTRP because it is the general case of the mTRP. For constrained NP-hard problem like the CmTRP, finding feasible solution is also NP-hard. The new contribution in this work is applied the VNS in both of two phases. The first phase generates feasible solution while the post one improve solution quality.

With respect to the instances, it indicates that the proposed algorithm can be used as follows:

- The fist way is to only run the construction phase with a rather loss of $8.7 \%$ solution quality on average. It is the fastest option.

- The second way is to run the construction phase and then enhance it by using one iteration. As the result, our algorithm obtains an average improvement of $6.35 \%$, and an average time of 0.5 seconds. It balances between solution quality and running time.

- The last is to run the construction phase and improve it no more than fifty iterations, and the average improvement reaches about $8.7 \%$, and an average time of 29.07 seconds. It is the best option in terms of solution quality.

Moreover, in comparison with some close variants of the mTRP, the proposed algorithm obtains the optimal solutions for the instances with up to 80 vertices in a short time. Moreover, it also reaches the better solutions than the algorithms in [1], [6] while it is comparable with the algorithms in [10], [12], [15], [16]. It shows that our algorithm is still effective for various problems.

\section{CONCLUSIONS}

In this paper, we propose a metaheuristic algorithm which applies the VNS in both of phases. The first phase creates a feasible solution while the post one improve it. The optimal solutions can be reached for the problem with up to 80 vertices in several seconds. The solution's quality is comparable with the previous algorithms for the other cases. Moreover, we give three options to use the proposed algorithm effectively. However, the running time needs to enhance to meet practical situations. It will be our aim in future research.

\section{ACKNOWLEDGMENT}

This research is funded by Hanoi University of Science and Technology (HUST) under grant number T2018-PC-208.

\section{REFERENCES}

[1] H.B. Ban, "An effective GRASP+VND metaheuristic for the kMinimum Latency Problem", Proc. RIVF, 2016, pp. 31-36.

[2] H.B. Ban, "General Variable Neighborhood Search for the QuoteTravelling Repairman Problem”, J. IJACSA, No. 4, 2020 (To appear).

[3] H.B. Ban, "A GRASP+VND Algorithm For The Multiple Traveling Repairman Problem With Distance Constraints", J. JCC, Vol.33, No.3, 2017, pp. 272-288.

[4] H.B. Ban, and D.N. Nguyen, "Improved genetic algorithm for Minimum Latency Problem”, Proc. SOICT, 2010, pp. 9-15.

[5] H.B. Ban, K. Nguyen, M.C. Ngo, and D.N. Nguyen, "An efficient exact algorithm for Minimum Latency Problem”, J. PI, No.10, 2013, pp. 1-8. 
[6] I. O. Ezzine, and Sonda Elloumi, "Polynomial Formulation and Heuristic Based Approach For The k-Travelling Repairman Problem", Int. J. Mathematics In Operational Research, Vol. 4, No. 5, 2012, pp. 503-514.

[7] F. Jittat, C. Harrelson, and S. Rao, "The k-Traveling Repairman Problem”, Proc. ACM-SIAM, 2003, pp.655-664.

[8] D. S. Johnson, and L. A. Mcgeoch, "The Traveling Salesman Problem: A Case Study In Local Optimization In Local Search In Combinatorial Optimization", E. Aarts and J. K. Lenstra, Eds., pp. 215-310.

[9] R. Jothi, and B. Raghavachari, "Minimum Latency Tours and The kTraveling Repairmen Problem", Proc. LATIN, 2004, pp. 423-433.

[10] L. Ke, and Z. Feng, "A Two-Phase Metaheuristic For The Cumulative Capacitated Vehicle Routing Problem", J. Computers and Operations Research, Vol. 40, No. 2, pp. 633-638, 2013.

[11] Y. Lua, U. Benlic, Q. Wua, and B. Peng, "Memetic algorithm for the multiple traveling repairman problem with profits", J. EAAI, Vol. 80 2019, pp. 35-47.

[12] Z. Luo, H. Qin, and A. Lim, "Branch-and-Price-and-Cut for The Multiple Traveling Repairman Problem With Distance Constraints", J. Operations Research, Vol. 234, No. 1, 2013, pp. 49-60.
[13] O. Martin, S. W. Otto, and E. W. Felten, "Large-Step Markov Chains For The Traveling Salesman Problem”, J. Complex Systems, Vol. 5, No. 3, 1991, pp. 299-326.

[14] N. Mladenovic, and P. Hansen, "Variable Neighborhood Search", J. Operations Research, Vol.24, No. 11 24, 1997, pp.1097-1100.

[15] SU. Ngueveu, C. Prins, and R. Wolfler Calvo, An Effective Memetic Algorithm for the Cumulative Capacitated Vehicle Routing Problem, J. Computers and Operations Research, Vol 37, No. 11, pp. 1877-1885, 2010.

[16] S. Nucamendi-Guillén, I. Martínez-Salazar, F. Angel-Bello, and J. M. Moreno-Vega, "A Mixed Integer Formulation and An Efficient Metaheuristic Procedure For The K-Travelling Repairmen Problem", J. JORS, Vol. 67, No. 8, 2016, pp. 1121-1134.

[17] A. Salehipour, K. Sorensen, P. Goos, and O.Braysy, "Efficient GRASP+VND and GRASP+VNS Metaheuristics for the Traveling Repairman Problem", J. Operations Research, Vol. 9, No. 2, 2011, pp.189-209.

[18] NEO (2013), http://neo.lcc.uma.es/vrp/vrp-instances/capacitated-vrpinstances. 